\title{
Análise da Vibração Mão-Braço na Colheita Florestal Semimecanizada
}

\author{
Luan Teixeira Mendes ${ }^{1}$, Nilton Cesar Fiedler ${ }^{*}$, Leandro Christo Berude ${ }^{1}$, Flavio Cipriano de \\ Assis do Carmo ${ }^{2}$, Ronie Silva Juvanhol ${ }^{3}$, Dâmaris Figueiredo Billo Nogueira ${ }^{1}$
}

\begin{abstract}
RESUMO: Operações semimecanizadas na colheita florestal podem trazer desconforto e riscos à segurança e à saúde dos operadores. Uma das máquinas muito utilizadas na operação de corte é a motosserra. Porém, seu uso diário pode acarretar graves acidentes e doenças devido à exposição a vibração, muitas vezes acima dos limites de referência das Normas Regulamentadoras. Este trabalho objetivou avaliar os níveis de vibração na operação de corte de madeira de eucalipto no município de Itabira, estado de Minas Gerais. A pesquisa foi realizada em um povoamento de Eucalyptus spp., com 14 trabalhadores, durante o período de abril a junho de 2017. A vibração foi mensurada utilizando o acelerômetro de acordo com as três coordenadas ortogonais (X, Y e Z) estabelecidas pela norma ISO 5349-1. De acordo com a classificação da exposição humana à vibração, foram observados que o traçamento e o desgalhamento tiveram sua classificação em fadiga em ambas coordenadas ( $\mathrm{x}, \mathrm{y}$ e $\mathrm{z}$ ).. Os resultados indicam que as motosserras utilizadas nas atividades avaliadas podem ocasionar danos à saúde e a integridade física dos operadores.
\end{abstract}

Palavras-chave: Técnicas e operações florestais; colheita semimecanizada; ergonomia florestal.

\section{Analysis of Hand-Arm Vibration in Semimecanized Forest Harvest}

ABSTRACT: The semi-mechanized operations in the forest harvest are generated by the discomfort and risks to the health and safety of the operators. One of the most used machines in the cutting operation is the chainsaw. However, its use is important for the Regulatory Norms. This work had priority in the operation indicators of the cutting operation of eucalyptus wood in the municipality of Itabira, state of Minas Gerais. The research was carried out in a settlement of Eucalyptus spp., During the period from April to June 2017. The vibration was measured using the accelerometer according to the three orthogonal guidelines (X, Y and Z), standards ISO 5349-1. According to the method of emission of a human sequence, the observed ones that traveled and diverted their categories in fatigue were both coordinates ( $\mathrm{x}$, $\mathrm{y}$ and $\mathrm{z}$ ). The results indicate that since chainsaws are used in the assessment areas they can cause damage to the health and physical health of operators.

Keywords: Forestry techniques and operations; semi-mechanized harvesting; forest ergonomics.

\section{INTRODUÇÃO}

A colheita florestal pode ser definida como um conjunto de operações realizadas no povoamento florestal, que compõe desde o preparo até a condução da madeira ao local de transporte, utilizando-se de técnicas e padrões preestabelecidos, com o intuito de transformá-la em produto final (LIMA E LEITE, 2014; SILVA et al., 2010). As operações de colheita florestal atingiram um estágio elevado de mecanização, porém algumas atividades, principalmente pequenos produtores, ainda necessitam do trabalho semimecanizado em virtude do alto custo de aquisição das máquinas e implementos, da baixa demanda e limitações devido à declividade do terreno.

As operações semimecanizadas quando conduzidas sem atender as normas vigentes podem ocasionar condições adversas ao bem-estar, à segurança e à saúde dos operadores. A exposição a vibrações oriundas das motosserras, máquina utilizada em sistemas semimecanizados, é um dos fatores mais preocupantes, pois o seu excesso torna a condição de trabalho desfavorável, causando desconforto, aumentando o risco de acidentes, podendo levar a danos consideráveis à saúde (FIEDLER et al., 2013).

Para acessorar na melhor adequação do trabalho ao homem, existe a ciência denominada ergonomia, que segundo Iida (2016) estuda o ser humano durante a execução de uma atividade ou trabalho, de forma a compreender as tarefas, os instrumentos, as máquinas e os sistemas de produção, visando melhor adequá-los a fim de proporcionar melhores condições de saúde, de segurança, e de bem-estar para trabalhador. De acordo com Alves et al. (2000), as avaliações ergonômicas contribuem expressivamente para que as condições de trabalho se tornem melhores, de modo a aumentar a qualidade de vida do trabalhador, que é uma condição essencial para o êxito de uma empresa ou empreendimento.

Segundo Fernandes (2000), um corpo é considerado em vibração a partir do momento que ele descreve um movimento oscilatório ao redor de um ponto de referência. A quantidade de ciclos completos de um movimento ao longo de um período de um segundo é denominada frequência e tem como unidade de medida Hertz [Hz].

Recebido em 16/07/2018; Aceito para publicação em 30/04/2019

${ }^{1}$ Universidade Federal do Espírito Santo

${ }^{2}$ Universidade Federal de Campina Grande

${ }^{3}$ Universidade Federal do Piauí

*E-mail: fiedler@pq.cnpq.br 
O resultado prejudicial das vibrações depende essencialmente dos seguintes fatores: pontos de aplicação no corpo, frequência das oscilações, aceleração das oscilações e duração da ação. O trabalhador está exposto de modo ativo à fonte de vibração através das mãos, nádegas, costas e pés. Por isso, a necessidade de realização de manutenções preventivas para evitar folgas entre as peças e também a necessidade de equipamentos mais sofisticados que eliminam ou minimizam as fontes de vibração no equipamento, garantindo os níveis de vibração em limites toleráveis.

O objetivo deste trabalho é avaliar os níveis vibração gerados pelas motosserras durante as atividades de corte florestal semimecanizado.

\section{MATERIAL E MÉTODOS}

Descrição da Área de Estudo

As atividades foram realizadas em uma propriedade produtora de madeira para fins de energia no município de Itabira, no estado de Minas Gerais. A região está localizada próxima às coordenadas geográficas $19^{\circ} 39^{\prime}$ 57" de latitude sul, $43^{\circ} 12^{\prime} 44^{\prime \prime}$ de longitude oeste. O relevo da região é montanhoso, com altitudes variando de $540 \mathrm{~m}$ na sede do município, a $1.662 \mathrm{~m}$ nos pontos mais altos.

Em Itabira, o clima é quente e temperado, classificado como Cwa de acordo com a Köppen e Geiger. A temperatura média anual é de $20,4{ }^{\circ} \mathrm{C}$, com pluviosidade média anual de $1.471 \mathrm{~mm}$, sendo o mês com menor precipitação, julho, com $9 \mathrm{~mm}$ e dezembro com maior precipitação, com uma média de $335 \mathrm{~mm}$.

Esse trabalho foi desenvolvido com dados levantados em uma propriedade rural, em um povoamento implantado em espaçamento inicial de $3 \mathrm{~m}$ x $2 \mathrm{~m}$ numa área de 6,3 hectares de Eucalyptus spp.

\section{Descrição da motosserra}

A motosserra utilizada nas operações de corte possui as especificações indicadas na Tabela 1:

Tabela 1. Dados Técnicos da motosserra avaliada

\begin{tabular}{lc}
\hline \multicolumn{1}{c}{ Parâmetro } & Valor \\
\hline Capacidade do tanque de combustível (l) & 0,685 \\
Cilindrada $\left(\mathrm{cm}^{3}\right)$ & 59 \\
Peso $(\mathrm{kg})$ & 5,6 \\
Potência (kW/cv) & $3,4 / 4,6$ \\
Rotação lenta (rpm) & 2,800 \\
Rotação máxima (rpm) & 14,000 \\
Vida útil (meses) & 14 \\
\hline
\end{tabular}

\section{Atividades Avaliadas}

O sistema de corte florestal analisado foi o semimecanizado com o uso da motosserra. Para a elaboração do trabalho, foi realizada uma caracterização detalhada das operações que compõem o corte florestal: derrubada, desgalhamento e traçamento/destopamento no sistema $1+1$ (um operador e um ajudante). Com cada dupla trabalhando com eitos de quatro linhas de plantio.

- Derrubada: Consiste na abertura de um entalhe direcional do lado da queda da árvore, com um corte horizontal e um obliquo, formando um angulo de $45^{\circ}$ graus, a uma altura de $10 \mathrm{~cm}$ do solo, no lado oposto da boca é feito o corte de queda horizontalmente a $15 \mathrm{~cm}$ de altura do solo, com profundidade de metade do tronco. Posteriormente é impulsionado o tombamento da árvore.

- Desgalhamento: É a retirada dos galhos remanescentes ao corte da árvore.

- Traçamento e destopamento: É a divisão do fuste em toras com tamanhos de 2 metros conforme a exigência e especificações do mercado.

\section{Análise estatística dos dados}

A pesquisa foi conduzida a partir de um levantamento da operação de corte verificado o número mínimo de amostras necessário para um erro de 5\%, conforme Conaw (1977):

$$
\mathrm{N}=\frac{\mathrm{t}^{2} \times \mathrm{s}^{2}}{\mathrm{e}^{2}}
$$

Em que:

$\mathrm{N}=$ número mínimo de amostra necessário; $\mathrm{t}=$ Coeficiente tabelado de T de Student; s = Desvio padrão; e = Erro admitido (5\% de significância);

Os dados de vibração foram analisados nas operações de derrubada, desgalhamento, traçamento/destopamento, e pausas, através da análise de variância utilizando o teste F, a $1 \%$ de significância. Nas análises que obtiveram diferenças significativas, foi empregado o teste de Tukey, a $1 \%$ de significância.

\section{Vibração}

Os níveis de vibração produzidos pela motosserra nas operações de corte foram obtidos com o uso de um acelerômetro e medidor de vibração portátil da marca TECKNIKAO e modelo NK300. 
A avaliação do nível de exposição baseou-se no cálculo do valor da exposição diária para um período de referência de 8 horas $(\mathrm{A}(8))$.

Com o objetivo de calcular a vibração nos eixos XY, foi utilizado a equação 2, definida na norma ISO 5349-1 e EU Good Pratice Guide HAV (2006) e observando as determinações da DIRECTIVE 2002/44/EC da União Europeia.

$$
A(8)=\sqrt{a^{2} h w x+a^{2} h w y}
$$

Em que $a^{2} h w x$ e $a^{2} h w y$ são os valores adquiridos para a palma da mão e nós dos dedos.

A Tabela 2 se refere aos valores aceitáveis de exposição à vibração nos eixos $\mathrm{x}, \mathrm{y}, \mathrm{z}$ segundo a Norma ISO 5349-1 (2001).

Tabela 2. Guia para avaliação da exposição humana a vibração.

\begin{tabular}{ccc}
\hline Critérios de Severidade & Aceleração $(\mathbf{X}, \mathbf{Y})$ horizontal $\left(\mathbf{m} / \mathbf{s}^{\mathbf{2}}\right)$ & Aceleração $(\mathbf{Z})$ vertical $\left(\mathbf{m} / \mathbf{s}^{\mathbf{2}}\right)$ \\
\hline Exposição & $0,224-0,448$ & $0,315 \dashv 0,630$ \\
Fadiga & $0,071 \dashv 0,224$ & $0,100 \nmid 0,315$ \\
Conforto & $<0,071$ & $<0,100$ \\
\hline
\end{tabular}

Fonte: ISO 5349-1 (2001).

A mensuração da vibração foi realizada adotando um sistema de coordenadas em três eixos, permitindo um valor da aceleração ponderada pela frequência, para os eixos $x, y, z$, representados por $a_{h w x}$, $a_{h w y}$ e $a_{h w z} e m ~ m / s^{2}$.

Combinando as três medidas, por intermédio da raiz da soma dos quadrados de cada elemento, foi obtida a vibração total para realização dos procedimentos estatísticos, respeitando as orientações da ISO 5349 -1 (2001) e EU Good Practice Guide HAV, (Equação 3).

$$
\mathrm{A}_{\mathrm{hv}}=\sqrt{\mathrm{Ahwx}^{2}+\mathrm{Ahwy}^{2}+\mathrm{Ahwz}^{2}}
$$

\section{RESULTADOS E DISCUSSÃO \\ Número mínimo de amostras}

O número mínimo de amostras foi respeitado para todas as atividades avaliadas de acordo com a Tabela 3. Deste modo teve-se à necessidade da uniformidade dos dados para a realização do teste estatístico adequado. Para as atividades analisadas, foram coletadas amostras piloto de medições de vibração. A vibração foi avaliada para cada atividade feita pelo operador, fazendo anotações correspondentes aos valores informados no instrumento de medição.

Tabela 3. Número mínimo de amostras para a necessária análise de vibração

\begin{tabular}{lcc}
\hline \multicolumn{1}{c}{ Atividade } & $\mathbf{N}^{\circ}$ mínimo de amostras & $\mathbf{N}^{\circ}$ de amostras coletadas \\
\hline Derrubada & 82 & 100 \\
Desgalhamento & 81 & 100 \\
Traçamento/destopamento & 67 & 100 \\
\hline
\end{tabular}

Vibração traçamento, pelo teste $\mathrm{F}$ ao nível de $1 \%$ de probabilidade.

Após realizar a análise estatística, foi observada a Os resultados da análise de variância e do Teste Tukey diferença significativa entre os níveis de vibração obtidos são evidenciados nas Tabelas 4 e 5 .

Tabela 5. Média de vibração nas operações e resultados do Teste Tukey.

\begin{tabular}{ll}
\hline \multicolumn{1}{c}{ Operações } & Médias* \\
\hline Derrubada & $0,2448 \mathrm{a}$ \\
Desgalhamento & $0,1965 \mathrm{~b}$ \\
Traçamento/Destopamento & $0,2763 \mathrm{c}$
\end{tabular}

Em que: As médias seguidas por uma mesma letra não diferem estatisticamente (Tukey, p > 0,05).

De acordo com a classificação da exposição humana a ambas coordenadas (xy e z). No entanto, a atividade de vibração e estabelecida pelas Normas ISO 5349-1, foram derrubada foi classificada em fadiga na coordenada z e observados que o traçamento/destopamento e o em exposição na coordenada xy, como mostrado na desgalhamento tiveram sua classificação em fadiga em Tabela 6. 
Tabela 6. Classificação da exposição humana a vibração conforme a ISO 5349-1

\begin{tabular}{lccc}
\hline \multicolumn{1}{c}{ Atividade } & Coordenadas & Aceleração $\left(\mathbf{m} / \mathbf{s}^{\mathbf{2}}\right)$ & Classificação \\
\hline \multirow{2}{*}{ Derrubada } & $\mathrm{xy}$ & 0,232 & Exposição \\
\cline { 2 - 4 } & $\mathrm{z}$ & 0,277 & Fadiga \\
\hline \multirow{2}{*}{ Desgalhamento } & $\mathrm{xy}$ & 0,157 & Fadiga \\
\hline \multirow{2}{*}{ Traçamento/Destopamento } & $\mathrm{z}$ & 0,196 & Fadiga \\
\cline { 2 - 4 } & $\mathrm{xy}$ & 0,197 & Fadiga \\
\hline
\end{tabular}

A legislação trabalhista adverte que os critérios de severidade à exposição da vibração classificados como conforto não devem atingir valores superiores a $0,071 \mathrm{~m} / \mathrm{s}^{2}$ para o eixo XY e $0,100 \mathrm{~m} / \mathrm{s}^{2}$ para o eixo Z. Mas como mostrados nos resultados, todos os valores de aceleração permaneceram acima desses limites. Dessa forma se classificando como fadiga ou exposição.

Com a finalidade de reduzir a vibração emitida pela motosserra deve ser realizadas manutenções e ajustes periódicos, substituindo peças gastas, avariadas e defeituosas. Segundo Leão (2005), para atenuar efeitos negativos da exposição a vibrações, deve se impedir a exposição contínua com pausas de 10 minutos por hora contínua de exposição.

\section{CONCLUSÕES}

Com o trabalho desenvolvido e as avaliações realizadas concluiu-se que, as motosserras utilizadas na operação de corte semimecanizado, colocam os operadores em riscos aos níveis de vibração.

Os níveis de vibração ultrapassaram os limites de referência da norma ISO 5349-1, atingindo a classificação de exposição no eixo xy da operação de derrubada e a classificação de fadiga nas operações de desgalhamento e traçamento.

Conforme a avaliação da exposição humana à vibração emitida ao sistema mão-braço, as condições e o tempo de exposição expõem os operadores a riscos e podem provocar danos à saúde.

\section{REFERÊNCIAS}

CONAW, P. L. Estatística. São Paulo: Edgard Blucher Ltda, 1977. 264 p.

DIRECTIVE 2002/44/CE. Prescrições mínimas de segurança e de saúde relativas a exposição dos trabalhadores aos riscos por agentes físicos (vibrações) do Parlamento Europeu. EU Good Practice Guide HAV; WBV Good practice Guide v. 6, 7 p., 2006.

FERNANDES, J.C. Segurança nas Vibrações sobre o Corpo Humano, 2000. Apostila

Fiedler, N. C; MARIN, H. B. ; OLIVEIRA, J. T. S. ; CARMO, F. C. A. . Análise da vibração no processamento secundário de madeiras com diferentes massas específicas aparentes. Floresta, v. 43, p. 1-8, 2013.

IIDA, I. Ergonomia: projeto e produção. São Paulo: Editora Edgar Blücher Ltda, 2016.

INTERNATIONAL ORGANIZATION FOR STANDARDIZATION. ISO 5349-1:2001. Mechanical vibration - Measurement and evaluation to human exposure to hand-transmitted vibration - Part 1 : General.

LEÃO, R. D. PERES, C. C. Noções sobre DORT, Lombalgia, Fadiga, Antropometia, Biomecânica e Concepção do Posto de Trabalho. 2005. Disponível em <http://www.celuloseonline.com.br/imagembank/>.

Acesso em 12 jun. 2017

LIMA, J. S. S.; LEITE, A. M. P. Mecanização. In: MACHADO, C. C. (Org.). Colheita florestal. Viçosa: Editora UFV, p. 43-73. 2014.

SILVA, E. N. et al. Avaliação técnica e econômica do corte mecanizado de Pinus sp. com Harvester. Revista Árvore, v.34, n.4,p. 745-753, 2010. 